\title{
Miranda
}

Revue pluridisciplinaire du monde anglophone /

Multidisciplinary peer-reviewed journal on the English-

speaking world

$20 \mid 2020$

Staging American Nights

« Voicing my desire to her ». Poétique du désir et coming-out littéraire dans les nouvelles « On Monday of Last Week » et « Grace »

\section{Sophie Okunhon}

\section{OpenEdition}

Journals

Édition électronique

URL : http://journals.openedition.org/miranda/26847

DOI : $10.4000 /$ miranda.26847

ISSN : 2108-6559

\section{Éditeur}

Université Toulouse - Jean Jaurès

\section{Référence électronique}

Sophie Okunhon, « « Voicing my desire to her ». Poétique du désir et coming-out littéraire dans les nouvelles « On Monday of Last Week » et « Grace » », Miranda [En ligne], 20 | 2020, mis en ligne le 20 avril 2020, consulté le 16 février 2021. URL : http://journals.openedition.org/miranda/26847 ; DOI : https://doi.org/10.4000/miranda.26847

Ce document a été généré automatiquement le 16 février 2021.

\section{c) (†)}

Miranda is licensed under a Creative Commons Attribution-NonCommercial-NoDerivatives 4.0 International License. 


\title{
«Voicing my desire to her ». Poétique du désir et coming-out littéraire dans les nouvelles « On Monday of Last Week » et « Grace »
}

\author{
Sophie Okunhon
}

\section{Introduction}

1 Généralement, lorsque les autrices féministes nigérianes expriment des luttes sociales et politiques, elles voient les hommes comme des alliés et donc dépeignent des couples hétérosexuels, excluant toutes représentations du désir lesbien (Azuah 131). Toutefois, des autrices nigérianes contemporaines remettent en question cette posture. Tout en critiquant l'Occident et l'économie patriarcale, Chimamanda Ngozi Adichie dans la nouvelle « On Monday of Last Week» (2009) et Chinelo Okparanta dans « Grace » (2013) présentent l'homosexualité féminine de façon décomplexée et modernisent de ce fait la littérature africaine postcoloniale féminine. Ainsi, ces autrices nigérianes empruntent le motif de la tentation érotique pour explorer, voire banaliser, un sujet tabou, un crime dans la société nigériane: celui du désir homosexuel féminin et de son accomplissement.

2 Adichie et Okparanta relatent différemment la surprenante découverte de l'homosexualité de leurs héroïnes. Alors que l'héroïne d'Adichie ressent une attirance mais ne succombe pas physiquement à la tentation, celles d'okparanta sortent du placard, transgressent tous les interdits (relation femme-femme, professeur-étudiant), expriment l'indicible, et franchissent le pas.

3 « On Monday of Last Week » retrace la naissance du désir lesbien de Kamara, Nigériane qui émigre aux États-Unis pour rejoindre son époux Tobeshi. Insatisfaite, car ne reconnaissant plus son mari, Kamara voit éclore en elle du désir pour Tracy, artiste noire-américaine et mère de Josh, l'enfant dont elle a la charge, qui la libère d'un 
mariage qui ne comble plus ses attentes: "She was finally with Tobeshi in America, finally with her good man, and the feeling was one of flatness » (Adichie 2017, 86). La focalisation interne adoptée par Adichie permet de suivre l'éveil de ce désir intime qui aboutit à une frustration - une frustration qui n'est que le résultat d'un désir non partagé.

4 "The first time I see her, she is crouched by the entrance of the third-floor bathroom, at an equal distance between my office and the lecture hall where I teach » (Okparanta 123). Dans ce qui ressemble à un journal intime manuscrit, Okparanta narre au présent par le truchement d'une narratrice homodiégétique la découverte de la bisexualité d'une professeur américaine de théologie. Divorcée, mise à l'écart par sa fille, la narratrice partage avec les lecteurs/lectrices ses souvenirs. Elle se remémore l'évolution de son attirance pour Grace - jeune doctorante nigériane-américaine de vingt-trois ans, le malaise, le conflit intérieur, l'(in)assouvissement du désir, et l'amour que Grace lui déclare. Son point de vue érige Grace en objet de désir. Le sujet tabou de la sexualité féminine et lesbienne ne peut donc être posé sans la dramatisation du rapport entre le sujet et l'objet de son désir.

Malgré une écriture de la tentation bien distincte dans son rapport à la transgression, la mise en scène du désir homosexuel, alliant description et parole performative, ouvre les textes à la fois vers une poétique de la séduction et vers une écriture politique libératrice du corps. Adichie et Okparanta se servent du motif du double, empruntent par touche des épisodes bibliques et articulent songeries et souvenirs pour exprimer ce désir-femme, un désir que rythme la rupture. Elles n'hésitent pas à jouer sur l'incompatibilité entre la raison et les sentiments, le corps et l'esprit, pour inventer à travers la tentation érotique une ligne de fuite, un «coming-out" possible entre la jouissance et l'angoisse, la culpabilité et la responsabilité, l'infamie et la morale religieuse. Pour qu'une libération ou une métamorphose du corps puisse s'opérer, elles remettent en question les attentes sociétales des relations genrées. L'écriture subversive est principalement présente dans « Grace ». Dans cette nouvelle, Okparanta balaie les valeurs religieuses traditionnelles par la relation amoureuse de ses personnages. Mais le triomphe d'un désir transgressif a un prix car narratrices et héroïnes doivent lutter contre leur désir. Cette lutte ajoute une dimension religieuse aux nouvelles : les héroïnes confessent leur désir interdit, à elles-mêmes, à une tierce personne et méta-textuellement aux lecteurs/lectrices. Ainsi, les textes explorent comment la conscience devient le site du paradoxe où désir et angoisse se rencontrent.

L'article consistera à analyser la manière dont les autrices rendent compte, dans leur texte, de la progression de l'inscription du corps des femmes nigérianes, prises aux pièges à la fois par leur propre raison et par les valeurs culturelles, sociétales ou religieuses, vers la réalisation d'une libération et d'une métamorphose de soi, que le désir soit assouvi, ou bien qu'il soit frustré. De quelle manière le désir marque-t-il le corps ? Comment ces deux autrices nigérianes articulent-elles une poétique du désir, de séduction et une écriture subversive dans un "coming-out " littéraire féminin et africain? Dans quelle mesure l'écriture du désir-femme met-elle en abyme la volonté des écrivaines de placer la femme au-devant de la scène? Il s'agira d'étudier le paradoxe du désir-femme à travers l'articulation de trois idées : le motif du double, le rôle de la religion et son incidence sur le désir, le triomphe paradoxal du désir ou l'ouverture sur l'intime par le biais de l'imagination. Il s'agira d'étudier, dans un deuxième temps, le rapport entre la transgression et l'écriture subversive. 


\section{Paradoxe du désir : tentation libératrice}

\section{Le motif du double}

7 Dans la nouvelle " On Monday of Last Week », le désir est rythmé par la présence de ruptures illustrées par les couples et par l'opposition entre Amérique et Afrique.

La première rupture est celle du couple américain. Les paroles de Neil concernant sa femme instaurent le doute: "Tracy is an artist. She spends a lot of time in the basement for now. She's working on a big thing, a commission. She has a deadline..." His voice trailed off " (Adichie 2017, 78). ${ }^{1}$ Tracy et Neil vivent dans deux mondes séparés. S'isolant dans cette maison-tanière (terme employé pour renvoyer à une pièce du rez-de-chaussée, « in the den» (79)), Tracy se réfugie au sous-sol pour son travail d'artiste et ne doit pas être dérangée; le père occupe le haut : le salon, la cuisine. La démarcation de l'espace pour chacun entrâne plusieurs hypothèses. Malgré les indices d'un amour réciproque passé, la présence de l'enfant et la photo posée sur une étagère dans leur tanière métaphore du foyer, il se peut que les partenaires de ce couple, indépendant l'un de l'autre, s'isolent pour ne pas perdre leur identité respective. La femme, Tracy, ne dépend pas de son époux; elle est une femme autosuffisante et libre. Il est également probable que Tracy et Neil vivent un ménage à trois - Tracy, Neil et une tierce personne, avec la nourrice Kamara ou la professeur de français Maren :

Neil [...] raising his voice so that Maren, in the den, would hear, [...] added, "You haven't met Maren, have you ?" Neil introduced them. Maren extended her hand and Tracy took it (94).

9 À moins qu'il ne s'agisse sinon d'une relation ouverte, où chacun vit sa sexualité de son côté. Une autre hypothèse révèle un couple en instance de divorce ou de séparation. Kamara se demande si Neil converse avec son épouse sur les besoins de leur fils. C'est le père qui s'interroge sur les besoins nutritifs de l'enfant, qui suit sa scolarité. La mère est absente. Les lecteurs/lectrices, tout comme la narratrice, ne peuvent s'empêcher de questionner les raisons des défaillances affectives de Tracy en tant que mère et épouse. L'absence de Tracy déclenche chez Kamara un sentiment de compassion envers cet enfant fragile :

"Why don't you lie down for a bit and watch a movie, Josh," she said. He liked to sit in the kitchen and watch her cook, but he looked so tired [...] (75).

Kamara watched Josh slot in a Rugrats DVD and lie down on the couch, a slight child with olive skin and tangled curls (75, c'est moi qui souligne).

Elle éprouve aussi de la pitié envers Neil ${ }^{2}$ et une curiosité grandissante pour Tracy:

At first Kamara was curious about this woman [...] Kamara wondered if and when Tracy left the basement. [...] She wondered whether Tracy ever saw her child (79).

11 Cette dernière déclenchera chez Kamara une «curiosité sexuelle » jusqu'alors ignorée (Phiri 159, ma traduction). Neil projette son anxiété due à la rupture de son mariage sur l'enfant en le tyrannisant par des inventions alimentaires. Ainsi, le corps de cet enfant se trouve à l'intersection de multiples désirs et sentiments :

He knew - he had to know - that the only reason she went into the bathroom each time she handed him the glass of green juice was to give him a chance to pour it away. It had started the first day Josh tasted it, made a face, and said, "Ugh. I hate it" (Adichie 2017, 74). 
Un jeu de miroir est omniprésent dans la dramatisation du désir. La désunion entre Neil et Tracy est mise en parallèle avec celle de Kamara et Tobeshi. Les vestiges d'un amour passé entre Tobeshi et Kamara sont conservés dans la mémoire de l'héroïne - mémoire qui se matérialise par l'anaphore du verbe "se souvenir » (84-85). À l'instar de Neil, Kamara, frustrée par une vie sexuelle sans flamme, cherche une solution à son insatisfaction. Au départ réticente, Kamara, diplômée, accepte de devenir nourrice pour échapper à une vie confinée avec Tobeshi pour soulager l'impression d'être écrouée dans une prison:

But even as she walked to the train station nursing her scratched dignity, she knew that she had not really needed to be persuaded. She wanted the job, any job; she wanted a reason to leave the apartment every day (79).

Elle s'occupe de Josh comme de son propre fils, accompagne Josh et son père à des compétitions d'orthographe, "Read-A-Thon » $(74,75,92)$. L'enfant devient son échappatoire. Afin d'atténuer une vie de couple sans désir et une frustration sexuelle, Kamara cherche à tomber enceinte :

She had taken to closing her eyes while Tobeshi was on top of her, willing herself to become pregnant, because if that did not shake her out of her dismay at least it would give her something to care about (86).

Par l'attitude de son héroïne, Adichie critique le postulat selon lequel la femme ne peut s'épanouir et se définir entièrement que dans les joies de la maternité (Munro 192), critique que l'on retrouve dans son manifeste Dear Ijeawele: "Be a full person. Motherhood is a glorious gift, but do not define yourself solely by motherhood" (Adichie 2018, 9)3. L'erreur de Kamara réside dans son désir de combler un vide par une grossesse car c'est ailleurs, à l'instar de la passion de Tracy pour l'art, qu'un individu s'épanouit.

15 La figure du double se répercute dans d'autres motifs. La narration met en parallèle deux espaces, le Nigéria et les Etats-Unis, à travers le corps de l'homme. L'homme désiré au Nigéria s'oppose à l'homme émigré aux Etats-Unis. Alors que Tobeshi était à la fois désiré, désirable et efféminé, il devient indésirable, voire repoussant. Méconnaissable, Tobeshi est métamorphosé ; Kamara a une vision déformée de son mari parce qu'elle ne se souvient plus des poils, composante apparente du corps masculin, sur les orteils de Tobeshi :

He took off his shoes when they got to the apartment and she looked at his toes, dark against the milk-colored linoleum of the kitchen floor, and noticed that they had sprouted hair. She did not remember his toes with hair (Adichie 2017, 84).

De même, ses paroles de mauvais goût, accrues par la contraction de verbes propre au registre familier vernaculaire, bien loin du langage amoureux et séducteur, se sont transformées en un langage grotesque et peu séduisant. Tobeshi qui exprime son désir sexuel de manière grossière provoque chez sa femme un sentiment de dégoût et d'irritation : "Tobeshi, this person who [...] had begun to talk in that false accent that made her want to slap his face. I wanna fuck you, I'm gonna fuck you » (Adichie 2017, 85) ${ }^{4}$. La critique d'Adichie envers la vie en Amérique se révèle donc par la comparaison que fait Kamara entre ce que son époux est devenu et le souvenir de leur relation au Nigéria : l'Amérique semble avoir effacé son charme nigérian plein de vie. Cette critique de la vie américaine est reprise dans la description de la nourriture, omniprésente dans la nouvelle. Le contraste entre la cuisine nigériane et la cuisine américaine est poignant. Tandis que la première est pleine, goûteuse - « soggy grilled meat with raw onions » (85), un plat que les lecteurs, séduits, pourraient presque savourer, voire 
mâcher, grâce à une recette stylistique dans laquelle Adichie combine allitérations, voyelles fermées/i://I/et voyelles ouvertes/a:/ / $\Lambda$ - la seconde est fade et aseptisée. Josh écœuré ne boit pas le jus préparé par Neil. Le rapport à la nourriture reflète l'état d'âme de l'hérö̈ne. Le mal-être causé par une «relation terne avec un Tobeshi américanisé " (Phiri 159, ma traduction) et une frustration d'un désir inatteignable obligent Kamara à s'abandonner au plaisir gustatif. La gourmandise l'aide à faire face au désenchantement de son couple tandis que son refus de manger de la banane plantain reflète l'envie de se distancer de son pays natal qui rejette l'homosexualité et d'atteindre son nouveau désir : Tracy.

17 Son attirance pour le corps de Tracy lui renvoie une image d'elle-même. L'attirance de Kamara pour Tracy est double. Il naît à la fois d'une attirance sexuelle et d'un manque. Souhaitant être désirée, Kamara modifie son apparence. Elle se tresse les cheveux, commence à se maquiller au point que Josh lui confère le rôle de mère de substitution : «Kamara, I'm glad we are family. Shabat Shalom» (Adichie 2017, 90). Son affection pour Josh s'accroît car il ressemble énormément à sa mère :

Josh grinned and she thought about the curve of his lips being exactly like that of Tracy's. [...] She looked down at his little head lowered before her, his hair in helpless curls, and she wanted to hug him very close (81).

18 La courbe des lèvres de Josh renvoie aux formes voluptueuses de Tracy, et le désir de Kamara pour Tracy est si pressant qu'elle souhaite serrer l'enfant entre ses bras. Le passage précité, la réflexion de Kamara ( A fellow woman who has the same thing as you have? », 80), ainsi que son désir soudain de perdre du poids en voyant Tracy (" [She] appeared, curvy in leggings and a tight sweater [...]. It was a strange moment. Their eyes held and suddenly Kamara wanted to lose weight and wear makeup again ", 79), sont plein d'ambiguïté et de sous-entendu. En évoquant les lèvres, le corps pulpeux, l'étreinte mais aussi les parties génitales de la femme par l'euphémisme " la même chose » - figure de style que reprendra Okparanta pour dire le désir - Adichie donne à son texte un caractère indéniablement sensuel. Cette sensualité est dissimulée derrière le corps de l'enfant car il est l'objet par lequel l'héroïne exprime implicitement son désir tabou. Comme l'observe Aretha Phiri, «l'expression silencieuse du désir fait partie de l'esthétique queer et reconstitue les conditions dans lesquelles les relations non-normatives sont refoulées" (Phiri 156) 5 . De plus, le pronom réflexif " herself » ainsi que le préfixe "self- » renvoient aux deux femmes en un effet de miroir. Alors que Kamara a besoin du regard d'autrui, surtout d'une relation intime, binaire, épanouissante pour se sentir entière (" what drew her was the way he [Tobeshi] looked at her with awed eyes, eyes that made her like herself » (Adichie 2017, 83, c'est moi qui souligne), Tracy n'a besoin de personne : "she's pretty much self-sufficient down there " (78, c'est moi qui souligne). Cette lecture corrobore l'hypothèse que tire Nathasha Distiller de l'ouvrage The Practice of Love: Lesbian Sexuality and Perverse Desire de Teresa de Lauretis selon laquelle la femme lesbienne cherche à vivre « une relation avec soimême ", qu'elle s'aime elle-même dans le corps de l'autre (Distiller 53). Kamara explore donc dans l'autosuffisance de Tracy une nouvelle version d'elle-même, un désir et un corps jusqu'alors inexplorés. L'attirance sexuelle en revanche se manifeste dès lors que la règle est transgressée.

C'est par le motif du double que se construit une poétique du désir dans «Happiness Like Water ». La narratrice, professeur de théologie, est constamment renvoyée à son propre corps, à son apparence, lorsque son regard se pose sur Grace. En s'autorisant à 
contempler l'étudiante qu'elle n'avait jamais vue auparavant, la narratrice ne peut s'empêcher de penser à elle mais aussi à elle-même. Le corps de Grace - sa jeunesse devient un miroir prospectif et rétrospectif reflétant à la fois ce que la narratrice fut jadis, ce qu'elle est aujourd'hui, et ce qu'elle désire posséder. Une certaine inhibition s'empare de la narratrice laissant entendre un désir pour l'autre femme et pour cette aura qu'elle dégage. Elle veut tendre le bras, toucher l'objet de son désir mais n'y arrive pas, et se retrouve pétrifiée, stupéfaite par cette jeune femme, tirée par une force invisible :

Her head is covered in thin black braids (Okparanta 124).

I catch a glimpse of her with the long black braids, sitting in the corner at the very back of the room (125).

I notice the way her braids hang down past her shoulders. Something about the way they move as she walks makes me want to reach out and touch them, but I remain where I am and watch her walk out of the room (126).

Les structures causatives, la répétition de la mention de la chevelure tressée indiquant l'africanité exotique, attirante et royale de cette jeune femme, ainsi que l'épistrophe du mot «room » soulignent l'insaisissabilité du corps et le pouvoir féminin. La musicalité du passage, présente par le rythme iambique et la réécriture des chansons Something des Beatles (1969) et Something about the Way You Look Tonight de Elton John (1997), qui traitent d'une relation amoureuse et d'un désir insaisissable, rendent ce désir-femme poétique. Ainsi, la tentation prend corps, se matérialise d'abord par l'ouïe et la musicalité du texte, puis par le regard. La corporéité de l'objet désiré se forme par une description verticale, du haut vers le bas, avec des pauses centrées sur des vêtements féminins attisant le désir :

I take in her face again - that startling combination of youth and old age. Her clothes are even an extension of that paradox : a white dress shirt, buttoned almost to the very top, prudishly, though I can see the outline of her bra from the white, diaphanous cotton. She has tucked the bottom of the blouse into the waistline of her greyish skirt. On her feet, she wears a pair of simple leather slippers (126).

\section{Le rôle de la religion et son incidence sur le désir}

21 L'étude du rôle de la religion, ainsi que les thèmes sous-jacents de la tentation et de l'interdit, sont essentiels dans l'analyse de l'écriture du désir des écrivaines. Neil interdit à Kamara de descendre dans le sous-sol où se trouve Tracy: " "Josh isn't allowed in the basement for now, so you can't go down there, either." Neil paused. "You have to make sure you don't bother her for anything whatsoever" " (Adichie 2017, 78), et, comme pour Eve, l'interdiction éveille en elle un désir jusqu'alors occulté. Le drame féminin de la tentation du jardin d'Eden se rejoue dans " On Monday of Last Week ». Adichie féminise le Mal, animalise le corps séduisant, voluptueux de Tracy. Ce corps sinueux, "curvy ", coloré, "paint-stained », au regard tortueux, «squinting ", faisant allusion au tentateur, le serpent, prend Kamara par surprise et la séduit: "suddenly Kamara wanted to lose weight and wear makeup again» (79, c'est moi qui souligne), laissant ainsi entendre que Tracy, cristallisant désir et tentation, reflète une division subjective à laquelle Kamara tente de se mesurer.

Ce désir-femme étant perçu par les Nigérians comme un péché, Okparanta emprunte des euphémismes pour exprimer l'indicible. Les pronoms employés par les deux protagonistes sont délibérément imprécis : 
"When something happens that makes the union no longer sacred, I believe that is grounds enough for divorce."

"But is the Bible okay with that?" she asks. "Is God okay with that?" (Okparanta 128, c'est moi qui souligne) exprimer leur désir. De plus, le métier de la narratrice - professeur de théologie ajoute la dimension de la confession. Son bureau se transforme en confessionnal :

On Thursday, I'm sitting in my office with my door cracked open, flipping through my stack of mail, when she knocks on the door. I invite her in, and she shuts the door behind her. They sometimes do, when what they have to talk to me about is personal (Okaparanta 126).

Alors que la narratrice joue le rôle de confesseur, lorsque Grace angoissée lui pose des questions concernant ce désir lesbien interdit, ce même désir naît en elle. Tout comme Grace, la narratrice confesse ce désir aux lecteurs/lectrices; sa confession débute d'abord sur un déni : «It couldn't possibly be inappropriate, being that I'm a woman, and she's a woman, and I'm probably older than her mother " (Okparanta 132). Les champs lexicaux du péché, de l'infirmité, de la souillure mettent en avant le crime d'une telle attirance sexuelle. Okparanta parle d'infirmité, «cripples», d'une «imperfection» (127), et Adichie d'une absurdité profane: «Tufia! What kind of foolishness is that?» (Adichie 2017, 80) - le désir-femme étant inconcevable pour toute femme dévote craignant Dieu. "La transgression perçue comme absurde" (Paki 16), comme une rupture avec le sensé, se retrouve dans l'exclamation igbo Tufia. Ainsi, cette interjection se traduisant par «Dieu m'en préserve » renvoie au péché. Cette faute se retrouve également dans le rappel des mains tachées de Tracy qui métaphoriquement représente une souillure visible.

La concrétisation du désir interdit est une chose impensable pour les Nigérianes Kamara et Grace mais aussi pour la professeur, comme le suggère la punition que s'imagine la narratrice d'okparanta :

The air is cold and feels as if it is pricking the skin on my cheeks. I imagine pins and needles, and thin, rusting metal wires, poking my skin. I tell myself that perhaps this is my punishment, for these new thoughts, these inappropriate desires (Okparanta 138).

Le corps doit souffrir ne serait-ce que pour avoir joui de telles pensées. La conscience du professeur et de Grace est scindée en deux : elles doivent jongler entre l'infâme et la moralité. Dès lors, la conscience est le site du drame humain où désir du corps et angoisse dictée par la raison entrent en conflit.

Grace et la professeur se servent des valeurs religieuses et de la raison comme solution au renoncement au désir. L'emprunt des versets de la Bible pose l'infranchissable et diabolise le désir lesbien. L'insistance sur l'irrationalité du désir et sur la diabolisation de cette attirance sexuelle souligne le conflit du corps et de l'âme qui s'opère sur le corps lui-même. Prenant cette tentation à bras le corps, l'âme est tourmentée, le corps se fait violence et s'abîme dans la douleur :

She is crouched by the entrance of the third-floor bathroom. [...] She is clutching a handbag to her chest, grasping it as if it is some kind of life support, and then all of a sudden she starts to bawl so hard that she seems to be gasping for air (Okparanta 123). 
protagoniste en sortant des toilettes fait son coming-out et est désemparée par ce qu'elle vient sûrement de s'avouer : l'attirance qu'elle éprouve pour les personnes du même sexe. Okparanta décrit ce tourment composé d'un sentiment de culpabilité envers l'objet désiré par le prisme de la chrétienté : la douleur physique, inscription charnelle de cette lutte interne, dépeint une victoire possible sur la tentation. La narratrice révèle ce tourment en s'adressant aux lecteurs/lectrices par des questions rhétoriques: «Dieu est-Il un dieu de guerre ou de paix?» (Okparanta 127, ma traduction) Elle signale des contradictions dans la Bible; c'est aux lecteurs/lectrices de trouver une réponse en fonction de leur opinion ou de leur position religieuse. Elle progresse cependant dans sa réflexion.

\section{L'imagination : le triomphe paradoxal du désir ou l'ouverture sur l'intime}

Il existe dans le texte une volonté de désarticuler le corps de l'esprit, en passant sous silence les appels du corps. Adichie insiste sur le rôle de la raison pour contenir toute tentation:

Her friend Chinwe would say if she ever told her. Tufia! What kind of foolishness is that? Kamara had been saying this to herself, too, since Monday of last week (Adichie 2017, 80).

Mais alors que la raison fait taire le désir, le corps quant à lui, possède son propre langage et révèle le désir caché. Ainsi, c'est par la manifestation des symptômes que les lecteurs/lectrices perçoivent dans les actes manqués des héroïnes "l'existence d'un désir jusqu'alors insoupçonné " (Estellon 150). La narratrice d'Okparanta laisse inconsciemment échapper son désir :

"Does it say something sweet?" I ask, and immediately I'm embarrassed by the question, because I realize that I'm not only hoping it does, but I'm also voicing my desire to her (Okparanta 130).

L'héroïne d'Adichie ne peut également s'empêcher de se tenir droite dans une position attirante lorsque Tracy s'approche - « Kamara sucked in her belly » (Adichie 2017, 89) - ou d'être plus souriante: "On Monday of last week, though, he had noticed the change in her. "You're bright today, Kam," he said as he hugged her» (86). L'acte manqué dévoilé saisit le corps dans un champ infini de possibilités et permet une mise en mots progressive des sentiments les plus profonds : " Her feet itched to go down the stairs, to knock on Tracy's door and offer something: coffee, a glass of water, a sandwich, herself » (91). Car si l'envie de descendre offrir un verre d'eau, un café ou un sandwich à Tracy semble au premier abord provenir d'un sentiment amical, le dernier mot, " herself », brise le refoulement et libère le corps. La nourriture se transforme et devient érotique car elle permet de mobiliser tous les sens. En cherchant ce qu'elle pourrait lui offrir, Kamara imagine être vue, touchée, goûtée par Tracy. Dans la poétique du désir, l'érotisation du corps se réalise non seulement sur son propre corps mais également dans la construction de l'autre : le corps de la femme désirée est décrit avec délectation. Kamara par exemple compare la peau de Tracy à du beurre de cacahuète (79) et partage avec les lecteurs/lectrices cette image qui transforme le corps en objet de plaisir.

Ainsi, c'est par ces actes manqués que le désir transgressif jaillit, sort de l'abîme et révèle « une nouvelle posture : celle de ne plus attendre, de franchir le pas» (Estellon 
151). La transgression s'établit avant tout par l'imagination avant qu'elle ne se solde par une action concrète. De ce fait, l'imagination se déploie et l'impossible devient possible. La poésie du désir redonne espoir et l'imagination fait vivre :

What happened in the kitchen that afternoon was a flowering of extravagant hope, because what now propelled her life was the thought that Tracy would come upstairs again (Adichie 2017, 80).

Le jeu de la séduction se dessine dans l'imaginaire; le corps s'y abandonne : que se passerait-il si... ? Une des fuites possibles pour éviter le châtiment tout en savourant le plaisir réside dans la projection imaginaire de sorte que le statut du désir-femme devient libératoire car les héroïnes de ces textes à travers leur imagination expriment leurs fantasmes. Kamara intériorise une vision de Tracy pour créer son monde imaginaire où, comme le souligne Phiri, les postures normatives genrées ou sexuelles seraient inexistantes (Phiri 160). Le rêve active la concrétisation du désir et libère le(s) corps puisque, comme le constate Phiri, la transformation de l'être - de la subjectivité de Kamara - se manifeste à la fois psychologiquement et physiquement (Phiri 160). C'est dans les toilettes devant des miroirs que l'héroïne d'Adichie érotise les mains de Tracy glissant sur son ventre ou bas-ventre :

Since Monday of last week, Kamara had begun to stand in front of mirrors. She would turn from side to side, examining her lumpy middle and imagining it flat as a book cover, and then she would close her eyes and imagine Tracy caressing it with those paint-stained fingers. She did so now in front of the bathroom mirror after she flushed (Adichie 2017, 74).

Kamara prend conscience de son corps et s'imagine valider par un mariage conventionnel l'attirance sexuelle qu'elle ressent:

Tracy's hand was still on her chin, slightly tilting her head up, and Kamara felt, first, like an adored little girl, and then like a bride. She smiled again. She was extremely aware of her body, of Tracy's eyes, of the space between them being so small, so very small (87).

À l'instar de la mouvance de la subjectivité de Kamara - de l'impossible à la possibilité d'un désir valide - la langue employée se précise. Le désir sexuel s'exprime d'abord par l'euphémisme "her lumpy middle» qui renvoie au ventre (selon Phiri) mais aussi à l'organe génital, puis progresse par une gradation d'étapes dans la vie d'une femme : de la jeune fille à la mariée. Ainsi, l'espace entre les deux corps - la distance entre le sujet et l'objet de son désir - se réduit, soulignant la possible validation d'un désir interdit.

Dans son analyse, Aretha Phiri avance synthétiquement en conclusion que la métaphore du placard à « résonance métaphysique » (Phiri 161) est omniprésente dans "On Monday of Last Week». En effet, l'évocation des toilettes reprise par Okparanta symbolise le lieu où ce désir lesbien se manifeste car c'est dans cette zone intime que Kamara - à la fois cachée du regard extérieur et face à elle-même par le reflet des miroirs - avoue tacitement son désir-femme. Son imagination et le reflet donnent corps à son désir, le rendent palpable et les toilettes - symbolisant son coming-out secret - révèlent aux lecteurs/lectrices l'attirance sexuelle de la protagoniste. Cette lecture de la symbolique des W.C. comme lieu d'émergence ou d'éveil sexuel converge avec l'étude que fait A. Phiri: l'imagination réveille chez Kamara «sa propre féminité »", " une conscience de soi », et évoque "un éveil sexuel en plein essor » (Phiri 160).

37 L'imagination seule ne peut suffire pour valider un désir qui ne peut être assouvi. C'est donc par le jeu de la séduction dans les gestes, dans la sonorité des mots, dans la parole 
performative ${ }^{6}$ que l'écart entre le corps et l'esprit se réduit et que le corps peut céder au désir :

"What does your name mean ? Am I saying it right ? Ka-mara?"

"Yes. It's a short form of Kamarachizuoroanyi : 'May God's Grace Be Sufficient for Us."

"It's beautiful, like music. Kamara, Kamara, Kamara."

Kamara imagined Tracy saying that again, this time in her ear, in a whisper. Kamara, Kamara, Kamara, she would say while their bodies swayed to the music of the name (Adichie 2017, 89).

Or, Kamara voit son rêve se dissiper par la réitération de sa rencontre avec une autre femme - Maren professeur particulier de Josh à laquelle Tracy pose la même question : «Have you ever been an artist's model?» $(87,94)$ - soulevant la question de la réciprocité du désir. Kamara construit son désir sur ses propres attentes, sa propre lecture des mots et des gestes de Tracy comme l'indiquent ses pensées : «Why haven't you come up since Monday of last week? Why have your eyes not lit up at seeing me?» (91) Kamara élabore une histoire définitive caricaturale fondée sur des stéréotypes : parce que Tracy n'embrasse pas son mari - « Kamara was pleased that Tracy did not kiss Neil, that they said "Hi, you" to each other as though they were brother and sister " (94) -, parce qu'elle lui demande de poser nue pour son travail d'artiste, Tracy doit ressentir un désir lesbien. Sa lecture nigériane du monde fondée sur une position hétéronormative - Tracy occupant le sous-sol, espace masculinisé par le désordre, pendant que Kamara occupe la cuisine «un espace traditionnellement féminin » (Phiri 161, ma traduction) - l'induit en erreur; le désir qu'elle éprouve n'est pas exclusif et binaire. Kamara ne prend pas en compte le polyamour dans sa lecture du couple de Neil et Tracy ou la possibilité d'une relation ouverte. Ainsi, la répétition de la séduction non seulement invalide ce désir construit sur un récit subjectif, mais oblige également Kamara à admettre sa bisexualité et donc à se redécouvrir.

Okparanta, quant à elle, autorise une évolution progressive du désir par l'imagination - d'un épanouissement imaginaire à un aboutissement du désir en inscrivant les étreintes imaginées de la narratrice : «I replace Nwafor with myself. I imagine kissing her, and I imagine her leaning into me » (Okparanta 149), puis le désir se réalise : « As I kiss her, I don't think of the practical things, like what this will mean for my job, the scandal it might cause, the shame it might bring " (150). En validant la sexualité de la narratrice et de Grace, l'autrice concrétise ce désir tabou. Cependant, ce désir-femme ne s'accomplit pas pleinement. Le corps désiré bien que rapproché par le baiser reste hors de portée. En effet, le mariage arrangé entre Grace et le Nigérian aura lieu, la mort pourra séparer Grace et la narratrice : " "But then," she whispers, "who's to say that I won't die first? Who's to say that you won't be the one burying me?" " (Okparanta 150). Ce désir validé n'est que passage ; il ne sera pas reconnu par la société : «Then I pull away, because the whole thing feels not quite like a celebration, something like unadorned acceptance, just a bit short of joyful » (Okparanta 150-151).

\section{Coming-out : écriture subversive pour positionner la femme au-devant de la scène}

En ajoutant un grain de mystère à son histoire, Adichie laisse aux lecteurs/lectrices le pouvoir de (re)construire l'histoire amoureuse de l'héroïne avec les éléments du texte dont ils/elles disposent : Neil et Tracy, mari et femme, vivent deux vies parallèles; le 
sous-sol dans lequel vit et travaille Tracy ne serait-il pas l'endroit caché pour ses amours féminines? Et quid du tableau aux couleurs vives et désordonnées qui pourrait être l'indice d'un programme politique ou un symbole LGBT : « In the basement, a wide painting leaned against the wall. [...] It looked like haphazard splashes of bright paint to her » (Adichie 2017, 88) ?

Okparanta engage une discussion avec ses lecteurs/lectrices, à l'instar de la narratriceprofesseur qui note au tableau des versets, rédige des notes sur la différence entre les lois apodictiques et les lois casuistes, ainsi qu'une réflexion sur la bonté lors de son cours magistral :

I jot down some Bible verses on the board, write some notes about apodictic law versus casuistic law, about Hammurabi's Code versus the Ten Commandments, about goodness for goodness' sake versus goodness with an eye to some type of reward or punishment (Okparanta 125).

Par ailleurs, Okparanta trouble les attentes des lecteurs/lectrices. Toutes les hypothèses faites à partir de quelques éléments sur l'origine de la professeur fluctuent sans cesse : la professeur aux yeux bleus - «"He had blue eyes, too," she continues. "Only not as beautiful as yours." " (Okparanta 132) - semble être une femme blanche or elle a une connaissance fine des traditions culturelles nigérianes : "And I imagine the wedding, her mama tinkering with the wedding attire, fussing with the wrappers, placing the jigida beads just so » (148-149). L'essentialisation du corps de la femme à partir d'une dimension sociale ou d'un postulat culturel est impossible : la narratrice peut donc être blanche, noire, métisse, ou tout autre. Okparanta sème le doute intentionnellement sur toutes projections occidentales afin de permettre une lecture plus fluide, plus ouverte aux possibilités : un couple mixte, nigérian et (afro)américain, entre les coutumes occidentales et les traditions nigérianes - «the traditional and the white » (Okparanta 142) - et un mariage méta-textuel géopolitique entre l'Occident et l'Afrique peuvent exister ; contrairement à la nouvelle « On Monday of Last Week » où domination et tension entre le Sud et le Nord (Phiri 161, Dunton 428) et rupture entre les rêves et la réalité d'une solidarité transnationale entre les Africains et les Afro-américains (Munro 191, 194) se font jour. Okparanta universalise la thématique du désir-femme, lie l'Afrique et l'Occident et écarte "une lecture univoque sur l'homosexualité » féminine (Macharia 2010) en évoquant un désir transnational validé par le baiser des protagonistes. Elle annule les perceptions africaines du désir-femme comme étant une réalité occidentale. L'heure n'est plus d'essentialiser ce désir par un prisme nationaliste ou continental, pour Chinelo Okparanta il s'agit maintenant de comprendre ce désir tabou d'un point de vue ontologique et personnel. De plus, le cadre situationnel est significatif. Les deux coming-outs se situent aux Etats-Unis, révélant une possible prudence des écrivaines car au Nigéria, depuis 2014, tout soutien ou encouragement à une union de même sexe est un délit passible de dix ans de prison (UN Human Rights, Munro 187). C'est donc de l'extérieur que Chinelo Okparanta poursuit son programme politique en donnant aux lecteurs/lectrices un tout autre scénario où triomphe la transgression : le baiser des deux femmes est décrit dans une prose lyrique. La fin de la nouvelle lorsque les personnages se tiennent la main revisite la chanson Can't Help Falling in Love d'Elvis Presley:

The river is still glowing in shades of silver and gold. Grace is sitting next to me, and I can't help thinking that perhaps the verge of joy is its own form of happiness (Okparanta 151, c'est moi qui souligne). 
La promesse d'une découverte excitante et jouissive, promesse de plaisir, tend vers une libération des corps et des codes. L'homosexualité perçue comme "abomination" (Okparanta 129) dans un pays où les choix, les gestes et les paroles sont dictés par les religions est un crime passible de dix à quatorze ans de prison. Okparanta interroge donc la loi de 2014 réprimant l'homosexualité par le thème du désir-femme. En intitulant sa nouvelle "Grace ", renvoyant à la fois à la théologie mais aussi à la douceur et à la beauté, l'autrice fait une réponse féministe moderne aux lois gouvernementales, aux diktats de la société patriarcale, aux contraintes religieuses et aux stéréotypes du genre. Par ce nom qui révèle l'amour, la compassion de Dieu aux infirmes et aux pécheurs ${ }^{7}$, elle pardonne à ses personnages leur péché supposé et leur donne l'autorisation de ressentir ce désir. L'autrice cite Lévitique $20: 13$, questionne ce que la Bible indique être une imperfection, une maladie (Phiri 154), une tare, un péché :

What about love your neighbor as yourself, and yet, God forbids the cripples from approaching his altar? What kind of God bans the very creatures he created from coming to Him just because of imperfections out of their control?

"If a man also lie[th] with mankind, as he lieth with a woman, both of them have committed an abomination : they shall surely be put to death."' [...] "Does this apply to females ?" She asks. "Is it also an abomination for women to lie with women?"

And I don't think of the Bible, of its verses about unnatural affections and abominations. Because it doesn't feel sinful to me. Because none of this is meant to be a challenge to God (Okparanta 127, 129, 150, c'est moi qui souligne).

Okparanta efface par le regard de la narratrice-professeur l'imperfection de Grace : "when I look up at her, I see something all-beautiful in her, something all-perfect, if there is such a thing" (148). L'autrice donne la permission à la narratrice de désirer une femme et valide ce désir par la temporalité du récit - l'utilisation du présent: " And I understand that she's giving me permission to feel a way that I'm not sure I want the permission to feel » (149). L'objet qu'offre plus tard Grace à la narratrice symbolise ce cadeau littéraire de l'écrivaine à la communauté LGBT : l'acceptation et la possibilité qu'un désir autre puisse exister.

Par son caractère transgressif, le désir lesbien implique une libération des relations genrées et « une dimension exploratoire des lois » (Estellon 153). Adichie et Okparanta démantèlent les relations hétéronormatives ainsi que toutes les attentes genrées. Le nom de Tobeshi - un nom attribué aux hommes et aux femmes - révèle l'orientation bisexuelle de Kamara car en épousant un homme qui ne se conformait pas aux attentes du masculin - « He was quiet, bookish, smallish » (Adichie 2017, 83) - elle dissimulait sa lesbianité. C'est la déception de retrouver un homme différent dont la masculinité est exagérée - « As he walked back toward her in his slightly baggy jeans and a T-shirt, the tangerine-colored sun behind him, she thought for a moment that he was somebody she did not know at all » (85) - et sa lecture erronée stéréotypée de Tracy qui raniment son désir-femme. De plus, Okparanta explore la loi dans des versets bibliques du Nouveau Testament, notamment le verset 1: 10 du livre de Timothée. ${ }^{8}$ Okparanta remet en cause l'inspiration divine des Saintes Écritures, les tourne en contes populaires et intègre l'amour lesbien dans un cadre dont il est initialement exclu :

She quotes me 2 Timothy: "All scripture is given inspiration by God, and is profitable for doctrine, for reproof, for correction, for instruction in righteousness." She asks me, how exactly do we know that God has inspired the Bible? (Okparanta 127). 
En écrivant l'homosexualité féminine «au sein d'un cadre hétérosexuel» (Phiri 161), Adichie et Okparanta mettent la femme en premier plan et font passer l'homme au second plan. Adichie tourne en ridicule l'Occident : les anxiétés et l'ultra-libéralisme de Neil sont risibles. Le machisme est parodié par le personnage de Tobeshi. L'image de l'homme nigérian idéal et le mariage hétérosexuel sont bafoués. Il n'y a aucune sensation (Okparanta 149), aucun désir par conséquent aucun bonheur dans le mariage hétérosexuel arrangé décrit par Okparanta ou dans les ménages hétérosexuels de la nouvelle d'Adichie. En écrivant le désir-femme, les autrices s'éloignent de la bienséance et de la respectabilité :

As I kiss her, I don't think of the practical things, like what this will mean for my job, the scandal it might cause, the shame it might bring. I don't think of how I will explain all this to my daughter, to her husband, how they will explain it to their son. I don't think of all the scandalous affairs that I've witnessed in my twenty years at the university. I don't think of my reaction to them, not that I've been one to condemn, but I don't think of my former disbelief at people - colleagues who, at such distinguished positions at the university, allowed fleeting romances between themselves and their students to interfere with their careers. I don't think of this as we kiss (Okparanta 150).

Elles sortent de l'économie patriarcale, du narcissisme phallique, par l'expression d'un désir-autre. Le désir-femme de ces personnages s'inscrit désormais autour de l'art : par l'artiste (Tracy, son œuvre et son corps) ou par la créativité littéraire. Grâce aux mots, Okparanta rend possible la déclaration d'amour de Grace :

She leaves where she is sitting on the bench, moves to crouch down in front of me. She is still holding her little box. She stoops on one knee and looks me in the eye and tells me how it's all wrong. [...] But that she's in love, and she's been trying to fight it, but she can't fight it any more (148).

Par l'entremise de Grace, l'autrice donne symboliquement un sexe à la narratrice pour que celle-ci puisse se l'approprier comme elle le souhaite :

She reaches inside the box and takes out a small round object in gold wrapping. She holds out the object in the space above my thighs. 'For you,' she says to me. 'A wedding favour,' she says (150).

En employant le présent narratif dans la nouvelle, Okparanta crée des subjectivités mouvantes plurielles et étaye la théorie de Raymond Williams selon laquelle l'utilisation du présent permet d'échapper aux normes structurelles car le présent toujours en mouvement et atemporel ne se fixe pas par rapport au passé :

In most description and analysis, culture and society are expressed in an habitual past tense. If the social is always past, in the sense that it always formed, we have to find other terms for the undeniable experience of the present: not only the temporal present, the realization of this and this instant, but the specificity of present being, the inalienably physical. [...] If the social is the fixed and explicit the known relationships, institutions, formations, positions - all that is present and moving, all that escapes or seems to escape from the fixed and explicit and the known, is grasped and defined as the personal : this, here, now, alive, 'subjective' (Williams 128).

Autrement dit, ces femmes fictives arrivent à trouver un bonheur par la créativité littéraire - bonheur éphémère car seule la littérature rend possible ce désir interdit : " "Happiness is like water," she says. "We're always trying to grab onto it, but it's always slipping between our fingers" "(Okparanta 144). Par conséquent, l'écriture d'Okparanta soutient l'hypothèse selon laquelle l'art aide la femme à se construire et à s'épanouir sans la présence obligatoire de l'homme. Car si la femme éprouve des 
difficultés à exprimer un désir lesbien, l'écrivaine en exprimant le désir-femme renouvelle le langage pour l'accompagner à se frayer une place dans la littérature (Distiller 53). Par conséquent, le songe de Kamara dans lequel Tracy caresse son ventre et son bas-ventre " plat comme une couverture de livre » (Adichie et Pracontal 2019, 1) relève également de cette hypothèse et met en abyme la volonté - même si prudente d'Adichie de placer le corps de la femme au-devant de la scène littéraire.

Les écrivaines positionnent la femme aux subjectivités mouvantes (noire, lesbienne, africaine), initialement à la marge, au-devant de la scène littéraire. Pour ce faire, elles jouent sur une dialectique du nouveau et de l'ancien. Adichie utilise le souvenir pour opposer l'ancienne Kamara à celle qui ressentira ce nouveau désir. Okparanta oppose l'Ancien Testament au Nouveau et illustre par des épisodes et des versets bibliques une évolution du désir. Si le Nouveau Testament apporte un espoir, un renouveau avec le plus grand commandement du Christ (Okparanta 130), Okparanta apporte une nouvelle approche à l'écriture du désir. En mettant en scène le désir de deux personnages qui choisissent d'avancer à contre-courant (Paki), son objectif est politique. La relation transgénérationnelle entre la professeur et l'étudiante a une fonction politique car si la professeur incarne une vision passée du féminisme, sa description de Grace et son désir envers la jeune étudiante suggèrent «une acceptation radicale du désir lesbien à l'avenir » (Osinubi 683). Unoma Azuah note que l'écriture du désir lesbien s'oppose nettement aux positions féministes nigérianes (Azuah 130) mais à travers le personnage de Grace, Okparanta perçoit à l'avenir une alliance entre la position féministe existante au Nigéria et une nouvelle position - «I take in her face again - that startling combination of youth and old age » (126) - un mélange entre une littérature ancienne et nouvelle (Green-Simms 142) pour qu'émerge une littérature féministe qui arrive à défendre les droits de toutes les femmes à leur corps et à leurs désirs (Osinubi 684).

\section{Conclusion}

Ainsi, malgré une écriture antinomique du désir transgressif ces deux autrices se rejoignent par ce désir qui reste inassouvi. Cet article rejoint l'analyse que fait Unoma Azuah sur la voix lesbienne dans la littérature nigériane. Elle note que « les dissonances illustrées par les personnages révèlent constamment que le rythme dans la littérature lesbienne est limité et hésitant, et qu'il y une crainte même parmi les écrivaines les plus courageuses » (Azuah 139, ma traduction). ${ }^{9}$ Les discours publics autour du lesbianisme n'étant encore qu'à une phase émergente ${ }^{10}$ au Nigéria, l'inassouvissement du désir reflète la prudence des écrivaines. Bien que la société nigériane ne soit pas encore prête à accepter ce désir, Adichie et Okparanta continuent dans la lignée de leurs consœurs. Rejetant tout discours moralisateur, elles mettent en avant et nomment ce désir-femme longtemps perçu comme absurde (Distiller 53) et questionnent toutes les attentes vis-à-vis de la masculinité et de la féminité afin d'articuler, dans un coming-out littéraire africain, une poétique du désir-femme délocalisé - un désir qui n'est plus perçu comme une invention occidentale venue de l'extérieur (Osinubi 684). 


\section{BIBLIOGRAPHIE}

"OHCHR | UN Human Rights Chief Denounces New Anti-Homosexuality Law in Nigeria." Office of the High Commissioner for Human Rights. Accessed 15 February 2020.

https://www.ohchr.org/EN/NewsEvents/Pages/DisplayNews.aspx?

NewsID=14169\&amp;LangID=E.

Adichie, Chimamanda Ngozi. "On Monday of Last Week." 2009. The Thing Around Your Neck. London: Fourth Estate, 2017. 74-94.

---. Dear Ijeawele: a Feminist Manifesto in Fifteen Suggestions. 2017. London : Fourth Estate, 2018.

Adichie, Chimamanda Ngozi, et de Pracontal, Mona. "Lundi de la semaine dernière. " Le tremblement précédé de Lundi de la semaine dernière. Paris : Gallimard. 2019.1-19.

Adichie, Chimamanda Ngozi, et Marguerite Capelle. Chère Ijeawele. Un manifeste pour une éducation féministe. Paris : Gallimard. 2017. Accessed 15 April 2020. https://www.numilog.com/LIVRES/ISBN/9782072721977.Livre?utm_source=PDF-excerpt

Azuah, Unoma. "The Emerging Lesbian Voice in Nigerian Feminist Literature." Matatu - Journal for African Culture and Society 29:1 (2005): 129-142.

Distiller, Natasha. "The (Im)Possibility of Lesbian Desire." Empowering Women for Gender Equity 22:63 (2005): 44-57.

Dunton, Chris. "'Wheyting Be Dat?': The Treatment of Homosexuality in African Literature" Research in African Literature 20:3 (1989): 422-448.

Estellon, Vincent. "Éloge de la transgression. Transgressions, folies du vivre ? De la marche vers l'envol. " Champ Psychosomatique 38:2 (2005) : 149-166.

Green-Simms, Lindsey. "The Emergent Queer: Homosexuality and Nigerian Fiction in the 21st Century." Research in African Literatures 47:2 (2016): 139-161.

John, Elton. "Something About The Way You Look Tonight." The Big Picture, Bernie Taupin, Chris Thomas, Mercury Rocket, 1997, track 6. Genius, 2020. Accessed 15 April 2020.

https://genius.com/Elton-john-something-about-the-way-you-look-tonight-lyrics

Macharia, Keguro. "Homophobia is not a Single Story." The Guardian. Wed. 26 May 2010. Accessed 16 April 2020

https://www.theguardian.com/commentisfree/2010/may/26/homophobia-africa-not-singlestory

Munro, Brenna M. "States of Emergence: Writing African Female Same-Sex Sexuality." Journal of Lesbian Studies 21:2 (2017): 186-203.

Okparanta, Chinelo. "Grace." Happiness, Like Water. New York: Mariner Books, 2013. 123-151.

Osinubi, Taiwo Adetunji. "The Promise of Lesbians in African Literary History." College Literature 45:4 (2018): 675-686.

Paki Sale, Rosine. "Transgression de la violence machiste et promotion de l'idéal féminin dans le roman africain." In L'Écriture de la transgression : viol, violence, violation dans la littérature africaine. Ed. Stéphane Amougou Ndi et Raphaël Ngwe. Paris : L'Harmattan, 2018. 13-30.

Phiri, Aretha. "Queer Subjectivities in J.M. Coetzee's Disgrace and Chimamanda Ngozi Adichie's 'On Monday of Last Week.'" Agenda 29:1 (2015): 155-63. 
Presley, Elvis. "Can't Help Falling in Love." Blue Hawaii, George David Weiss, Luigi Creatore, Hugo Peretti, Radio Recorders, 1961, track 5. Genius,2020. Accessed 16 April 2020.

https://genius.com/Elvis-presley-cant-help-falling-in-love-lyrics

The Beatles. "Something." Abbey Road, George Harrison, Paul McCartney, Billy Preston, Ringo Starr, John Lennon, George Martin, Abbey Road Studios, 1969, track 2. Genius, 2020 Accessed 15 April 2020.

https://genius.com/The-beatles-something-lyrics

Williams, Raymond. Marxism and Literature. Oxford: Oxford University Press, 1977.

\section{NOTES}

1. « " - Tracy est artiste. Elle passe beaucoup de temps au sous-sol en ce moment. Elle travaille sur un gros projet, une commande. Elle a une date limite..." Il laissa sa phrase en suspens » (Adichie et Pracontal 2019, 4).

2. "Three months of listening to Neil's worries, of carrying out Neil's anxiety-driven instructions, of developing a pitying affection for Neil» (Adichie 2017, 79). Voir aussi la traduction professionnelle : « Trois mois à écouter les inquiétudes de Neil, à suivre les consignes nées de ses angoisses, à se prendre peu à peu d'une affection mêlée de pitié pour lui » (Adichie et Pracontal 2019, 5).

3. « Sois une personne pleine et entière. La maternité est un magnifique cadeau, mais ne te définis pas uniquement par le fait d'être mère " (Adichie et Capelle 2017, 18). Ce manifeste est traduit de l'anglais par Marguerite Capelle.

4. « Tobeshi, cet homme qui [...] s'était mis à parler avec un faux accent qui lui donnait envie de le gifler. I wanna fuck you. I'm gonna fuck you. J'ai envie de te baiser. Je vais te baiser " (Adichie et Pracontal 2019, 11).

5. Ce concept, traduit ici, est employé par Aretha Phiri dans son article : « Adichie's short story OMLW deploys silence as an "aesthetic of queerness" that on the surface appears to reconstitute the terms by which non-normative sexualities are marginalised, oppressed,and repressed» (Phiri 156).

6. Kamara désire obtempérer à l'injonction de Tracy : " "You will take your clothes off for me," she said. "Yes" " (Adichie 2017, 92). Les paroles sont performatives pour Kamara car elle retient son ventre s'imaginant déjà à moitié nue et plus désirable avec un ventre plat.

7. L'étymologie du nom provient du site Christianity.com https://www.christianity.com/theology/what-is-grace.html

8. Extrait du Livre de Timothée 1:8-11: « Nous n'ignorons pas que la loi est bonne, pourvu qu'on en fasse un usage légitime, sachant bien que la loi n'est pas faite pour le juste, mais pour les méchants et les rebelles, les impies et les pécheurs, les irréligieux et les profanes, les parricides, les meurtriers, les impudiques, les infâmes, les voleurs d'hommes, les menteurs, les parjures, et tout ce qui est contraire à la saine doctrine, conformément à l'Evangile de la gloire du Dieu bienheureux, Evangile qui m'a été confié ».

https://saintebible.com/lsg/1_timothy/1.htm

9. "The inconsistencies demonstrated by the characters constantly reveal that the pulse in lesbian literature is constricted and unsure, and that there is fear among even the bravest writers » (Azuah 139).

10. Le concept d'émergence théorisé par Raymond Williams exprime un processus qui s'oppose et résiste à tous les discours dominants ou hégémoniques. Selon Lyndsey Green-Simms, les œuvres des écrivaines nigérianes contemporaines s'inscrivent dans ce concept car leur écriture résiste 
aux discours dominants de manière inédite et raconte le désir homosexuel ou lesbien sans contenir de dimension moralisatrice (Green-Simms 142).

\section{RÉSUMÉS}

L'article analyse l'écriture du désir lesbien dans deux nouvelles de Chimamanda Ngozi Adichie et de Chinelo Okparanta, et le rapport entre la transgression et l'écriture subversive. L'articulation du désir-femme se fait autour de trois idées : le corps désiré et sa construction, le rôle de la religion et son incidence sur le désir, et l'ouverture sur l'intime par le biais de l'imagination. Par leur prudence, révélée par un triomphe paradoxal du désir-femme (in) validé des personnages, ces écrivaines s'inscrivent dans le concept d'émergence, ou ce que l'on peut nommer un coming-out littéraire, car elles résistent aux discours dominants de manière inédite. Rejetant tout caractère moralisateur, elles nomment ce désir-femme longtemps perçu comme absurde et questionnent toutes attentes genrées et sexuelles afin d'articuler, dans une littérature féministe nouvelle, le droit des femmes à disposer de leur corps et à assouvir leurs désirs. Par conséquent, l'écriture du désir interdit met en abyme leur volonté de placer la femme au-devant de la scène littéraire.

The article analyzes the writing of lesbian desire in two short stories by Chimamanda Ngozi Adichie and Chinelo Okparanta, and the connection between transgression and subversive writing. The articulation of female same-sex desire hinges on three ideas : the desired body and its construction, the role of religion and its repercussion on desire, and the intimate through the workings of imagination. By their prudence, revealed by a paradoxical triumph of the female characters' (in)validated same-sex desire, the writers are in keeping with the concept of emergence, or what we call a coming-out narrative since they unprecedentedly resist dominant discourses. Rejecting all moralizing aspects, they name this female same-sex desire otherwise perceived as absurd and question gender and sexual premises to articulate, in a new feminist literature, the right of women to own their bodies and to fulfill their desires. Consequently, writing forbidden desire mirrors the writers' wish to place women at the forefront of the literary scene.

\section{INDEX}

Mots-clés : désir, écriture subversive, transgression, coming-out littéraire

Keywords : desire, subversive writing, transgression, coming-out narrative

\section{AUTEURS}

\section{SOPHIE OKUNHON}

Doctorante sous la direction de Claudine Raynaud

EMMA - Études Montpelliéraines du Monde Anglophone EA741, Université Paul Valéry

Montpellier 3

sophie.okunhon@etu.univ-montp3.fr 